\title{
Effect of a Magnetic Field on Tunneling Conductance in Normal Metal d-Wave Superconductor Interfaces
}

\author{
William J. Herrera and J. Virgilio Niño \\ Departamento de Física, Universidad Nacional de Colombia, Bogotá- Colombia
}

Received on 8 December, 2005

\begin{abstract}
Through the analytic solutions of the Bogoliubov de Gennes (BdG) equations the effect of a static and homogeneous magnetic field applied parallel to the interface of an NIS (N: Normal metal, S: superconductor and I: Insulator) junction on the differential conductance is calculated. For a $d_{x y}$ - symmetry we obtain zero bias conductance peak that can be split by a magnetic field. The shift of the zero bias conductance peak depends on the spread $(\beta)$ of the tunneling electrons in $k$ space, on the magnitude of the applied field $H$ and on the ratio between the Fermi energy of the superconductor and the normal region, $E_{F S} / E_{F N}$. Finally we estimate the minimum value of the magnetic field, $H_{\min }$, that splits the zero bias conductance peak. In general $H_{\min }$ depends on $\beta, E_{F S} / E_{F N}$, the strength of the insulating barrier $Z$ and on the temperature $T$.
\end{abstract}

Keywords: Zero bias conductance peak; d-wave superconductivity; Magnetic field

\section{INTRODUCTION}

In high temperature superconductivity different experiments have been interpreted assuming a $d$-wave symmetry of the pair potential, see for example [1]. A feature of $d$ symmetry is the zero-bias conductance peak (ZBCP) observed in tunneling conductance in NIS junctions [1]-[6]. The ZBCP appears when the angle between the $a$ axis of the superconductor and the vector normal to the interface is nonzero; it is a maximum when this angle is $\pm \pi / 4,(110)$ orientation. This peak is due to the formation of the zero energy states (ZES) that are originated by the Andreev reflection at the interface; it undergoes a difference in phase of $\pi$ due to the anisotropy of the pair potential [7]-[10]. Different experiments have shown that the ZBCP can be split due to application of a magnetic field [2]-[5]. From numerical solutions of the Eilenberger's equations, it has been shown that the effect of the magnetic field is to produce a shift of the ZES proportional to the applied magnetic field [11]-[12], other experiments show [13][14] that the effect of the magnetic field is to decrease of the heigth of the ZBCP. In this work we solve the BdG equations for this system and show that the splitting depends on $Z, \beta, \chi$ and $T$, The above mentioned experimental characteristics are explained in this work.

\section{THEORY}

The quasiparticles in a superconductor are described by the BdG equations. For steady states and anisotropic superconductors these equations are [15]

$$
\begin{aligned}
& \int d \mathbf{r}_{2} \hat{H}\left(\mathbf{r}_{1}, \mathbf{r}_{2}\right) \psi\left(\mathbf{r}_{2}\right)=E \psi\left(\mathbf{r}_{1}\right), \psi\left(\mathbf{r}_{1}\right)=\left(\begin{array}{c}
u\left(\mathbf{r}_{1}\right) \\
v\left(\mathbf{r}_{1}\right)
\end{array}\right) \\
& \hat{H}\left(\mathbf{r}_{1}, \mathbf{r}_{2}\right)=\left(\begin{array}{cc}
H_{e}\left(\mathbf{r}_{1}\right) \delta\left(\mathbf{r}_{1}-\mathbf{r}_{2}\right) & \tilde{\Delta}\left(\mathbf{r}_{1}, \mathbf{r}_{2}\right) \\
\tilde{\Delta}^{*}\left(\mathbf{r}_{1}, \mathbf{r}_{2}\right) & -H_{e}^{*}\left(\mathbf{r}_{1}\right) \delta\left(\mathbf{r}_{1}-\mathbf{r}_{2}\right)
\end{array}\right),
\end{aligned}
$$

where $H_{e}\left(\mathbf{r}_{1}\right)=\left(-i \hbar \nabla-e \mathbf{A}\left(\mathbf{r}_{1}\right)\right)^{2} / 2 m+V\left(\mathbf{r}_{1}\right)-\mu$ is an electronic hamiltonian, with $\mathbf{A}\left(\mathbf{r}_{\mathbf{1}}\right)$ the vector potential associated with the magnetic fields present in the system, $V\left(\mathbf{r}_{\mathbf{1}}\right)$ the scalar potential and $\mu$ the chemical potential, $\tilde{\Delta}\left(\mathbf{r}_{1}, \mathbf{r}_{2}\right)$ is the pair potential, $u\left(\mathbf{r}_{1}\right)$ and $v\left(\mathbf{r}_{1}\right)$ are the wave function for the electronand hole-like components of a quasiparticle. The insulating barrier of height $V_{0}$ and thickness $d$ is located in $x>0$, this barrier can be modeled by a delta function, $V(x)=U_{0} \delta(x)$, where $U_{0}=V_{0} d$. We concentrate on cuprate superconductor junctions. It is supposed that the quasiparticle moves on the $\mathrm{CuO}_{2}$ plane with the $a$ and $b$ axes in the $x-y$ plane, the interfaces are normal to the $x$-axis, see Fig. 1. The Fourier transform of the pair potential is modeled by

$$
\Delta_{F T}(\mathbf{k}, \mathbf{r})=\int d \mathbf{R} \mathbf{e}^{i \mathbf{k} \cdot \mathbf{R}} \tilde{\Delta}\left(\mathbf{r}_{1}, \mathbf{r}_{2}\right) \simeq \Delta(\hat{\mathbf{k}}) \Theta(x),
$$

where $\mathbf{R}=\mathbf{r}_{1}-\mathbf{r}_{2}, \mathbf{r}=\left(\mathbf{r}_{1}+\mathbf{r}_{2}\right) / 2, \Theta(x)$ the Heaviside function and $\Delta(\hat{\mathbf{k}})$ is the pair potential that undergoes a quasiparticle with momentum $\mathbf{k}$, for $s$-symmetry $\Delta(\hat{\mathbf{k}})=\Delta_{0}$ and for $d_{x-y}$-symmetry $\Delta(\hat{\mathbf{k}})=\Delta_{0} \sin 2 \theta_{s}$, where $\theta_{s}$ is the quasiparticle angle in the superconductor region $\theta_{s}=\sin ^{-1}\left(k_{y} /|\mathbf{k}|\right)$. The magnetic field $H$, is applied parallel to the z-axis, therefore the vector potential can be written as $\mathbf{A}(\mathbf{r})=A_{y}(x) \hat{\mathbf{j}}$. We consider the situation of a high Tc superconductor where the coherence length of the pair potential $\xi$ is much smaller than the penetration length $\lambda$ of the magnetic field and approximate $A_{y}=-H \lambda$. As the potentials depends only on $x$, the solutions of the BdG equations can be written as $\psi(\mathbf{r})=e^{i k_{y} y} \tilde{\psi}(\mathbf{r})$.

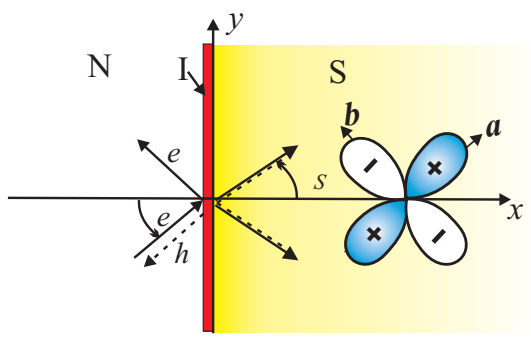

FIG. 1: NIS junction, The insulating barrier is located in $x=0$ and is modeled by a delta function. For $\mathrm{d}_{x y}$-symmetry, the pair potential is modeled as $\Delta\left(\theta_{S}\right)=\Delta_{0} \sin \left(2 \theta_{S}\right)$. The solid and dashed lines represent the electron and the hole-like components of a quasiparticle, respectively.

Considering an incoming electron from the normal region, 
the wave functions $\tilde{u}(x)$ and $\tilde{v}(x)$ are given taking into account the in the Andreev approximation [15] by

$$
\begin{aligned}
& \left(\begin{array}{l}
u(x) \\
v(x)
\end{array}\right)=\left(e^{i \tilde{k}_{+} x}\left(\begin{array}{l}
1 \\
0
\end{array}\right)+a e^{i \tilde{k}_{-} x}\left(\begin{array}{l}
0 \\
1
\end{array}\right)+b e^{-i \tilde{k}_{+} x}\left(\begin{array}{l}
1 \\
0
\end{array}\right)\right) \Theta(-x) \\
& +\left(C e^{i \tilde{k}_{+}^{+} x}\left(\begin{array}{c}
u_{0}^{+} \\
v_{0}^{+} e^{-i \varphi_{+}}
\end{array}\right)+D e^{-i \tilde{k}_{-}^{-} x}\left(\begin{array}{c}
v_{0}^{-} \\
u_{0}^{-} e^{-i \varphi_{-}}
\end{array}\right)\right) \Theta(x),
\end{aligned}
$$

where

$$
\begin{aligned}
& \tilde{k}_{ \pm}=\sqrt{k_{F N x}^{2} \pm 2 m \tilde{E} / \hbar^{2}}, k_{F N x}=\sqrt{k_{F N}^{2}-k_{y}^{2}}, \\
& \tilde{k}_{ \pm}^{ \pm}=\sqrt{k_{F S x}^{2} \pm 2 m \tilde{\Omega_{ \pm}} / \hbar^{2}}, k_{F S x}=\sqrt{k_{F S}^{2}-k_{y}^{2}}, \\
& \tilde{u}_{0}^{ \pm}=\sqrt{\frac{1+\tilde{\Omega}_{ \pm} / \tilde{E}}{2}}, \tilde{v}_{0}^{ \pm}=\sqrt{\frac{1-\tilde{\Omega}_{ \pm} / \tilde{E}}{2}}, \text { if } E>V_{A} \\
& \tilde{u}_{0}^{ \pm}=-\sqrt{\frac{1-\tilde{\Omega}_{ \pm}^{*} / \tilde{E}}{2}}, \tilde{v}_{0}^{ \pm}=\sqrt{\frac{1+\tilde{\Omega}_{ \pm}^{*} / \tilde{E}}{2}}, \text { if } E<V_{A}, \\
& \tilde{\Omega}_{ \pm}=\sqrt{\tilde{E}-\left|\Delta_{ \pm}\right|^{2}}, \tilde{E}=E-V_{A}, V_{A}=\frac{e \hbar k_{y} \lambda H}{m} .
\end{aligned}
$$

The wavenumbers $k_{F N}$ and $k_{F S}$ are determined by the Fermi energy in the normal and superconducting regions respectively, $k_{F S}^{2} / k_{F N}^{2}=E_{F S} / E_{F N}=\chi^{2}$. The quasiparticles with $k_{+}^{+}$ and $k_{-}^{-}$wavenumber move in the pair potential $\Delta_{+}$and $\Delta_{-}$ respectively

$$
\Delta_{ \pm}\left(\theta_{s}\right)=\Delta\left( \pm k_{ \pm}^{ \pm} \hat{\imath}+k_{y} \hat{\mathbf{j}}\right) \equiv \Delta_{ \pm} e^{i \varphi_{ \pm}} .
$$

The effect of the magnetic field is an energy shift that depends on $k_{y}$ and $H$. One finds $a, b, C$ and $D$ using the boundary conditions in $x=0$. The electron-electron and electron-hole reflection coefficients are respectively, $R_{e}=|b|^{2}, \quad R_{h}=|a|^{2}$.

\section{DIFFERENTIAL CONDUCTANCE}

Using the model developed by Blonder et al.[16] the differential conductance for an angle $\theta_{S}$ and for $T=0 \mathrm{~K}$ is calculated from $R_{e}$ and $R_{h}$ coefficients as

$$
\begin{aligned}
\sigma_{S}\left(e V, \theta_{S}\right) & =\sigma_{N}\left(\theta_{S}\right)\left(1+Z^{2}\right)\left(1+R_{h}\left(e V, \theta_{S}\right)-R_{e}\left(e V, \theta_{S}\right)\right) \\
& =\sigma_{N} \frac{1+T_{N} \tilde{\Gamma}_{+}^{2}+\left(T_{N}-1\right)\left|\tilde{\Gamma}_{+}^{2} \tilde{\Gamma}_{-}^{2}\right|}{\mid 1-\left(T_{N}-1\right) \tilde{\Gamma}_{+} \tilde{\Gamma}_{-} e^{-\left.i\left(\varphi_{+}-\varphi_{-}\right)\right|^{2}}}
\end{aligned}
$$

with

$$
\begin{gathered}
\tilde{\Gamma}_{ \pm}=\frac{\tilde{v}_{0}^{ \pm}}{\tilde{u}_{0}^{ \pm}}, \chi=\frac{k_{F S}}{k_{F N}}, Z=\frac{m U_{0}}{\hbar^{2} k_{F N}}, \\
T_{N}=\frac{4 \chi \cos \theta_{N} \cos \theta_{S}}{\left(\cos \theta_{N}+\chi \cos \theta_{S}\right)^{2}+4 Z^{2}},
\end{gathered}
$$

$\sigma_{N}$ is the differential conductance when $\Delta=0$ (NIN junction), $Z$ is the strength of the barrier and $\theta_{N}$ is the quasiparticle angle in the normal region and is determined by the momentum conservation condition in $y$ direction

$$
\sin \theta_{N}=\chi \sin \theta_{S}
$$

Firstly the case of $\chi=1\left(\theta_{N}=\theta_{S} \equiv \theta\right)$ is analyzed. The relative total differential conductance is found by integration in the $k$-space as

$$
\sigma_{R}(e V)=\frac{\left\langle\sigma_{S}(e V, \theta)\right\rangle}{\left\langle\sigma_{N}(\theta)\right\rangle}
$$

where

$$
\langle\ldots\rangle=\frac{\int_{-\pi / 2}^{\pi / 2} d \theta \cos \theta e^{-\beta \sin ^{2} \theta} \ldots}{\int_{-\pi / 2}^{\pi / 2} d \theta \cos \theta e^{-\beta \sin ^{2} \theta}}
$$

and $\beta$ is related to the spread of tunneling electron in $k$ - space, it is given by

$$
\beta=\hbar d k_{F}^{2} / \sqrt{2 m V_{0}}
$$

It is important to note that the insulating barrier is characterized by the parameter $Z$ and $\beta$. If in the equation (6) $H=0$, our results agree with [7]-[8]. When $H \neq 0$ the differential conductance $\sigma_{S}(e V, \theta)$ has a shift given by $V_{A}(\theta)$. The Gaussian distribution, $e^{-\beta \sin ^{2} \theta}$, diminishes $99 \%$ for a angle given by $\theta_{c}=\sqrt{\ln (100) / \beta}$. The maximum peak energy shift is determined by $V_{A}\left(\theta_{\max }\right)$, where $\theta_{\max }$ is the maximum angle for which an electron tunnels the insulating barrier. This angle is $\pi / 2-\varepsilon(\varepsilon \rightarrow 0)$ if $0<\beta \leq 4.2$ or approximately $\theta_{c}$ if $\beta>4.2$.

$$
V_{A, \max }=e \hbar k_{F} \sin \theta_{\max } \lambda H / m \text {. }
$$

The maximum value of $V_{A}$ is $\max \{\Delta(\theta)\}=\Delta_{0}$, therefore the maximum magnetic field is

$$
H_{\max }=H_{c} / \sin \theta_{\max },
$$

with $H_{c}$ the bulk critic magnetic field. The average shift is

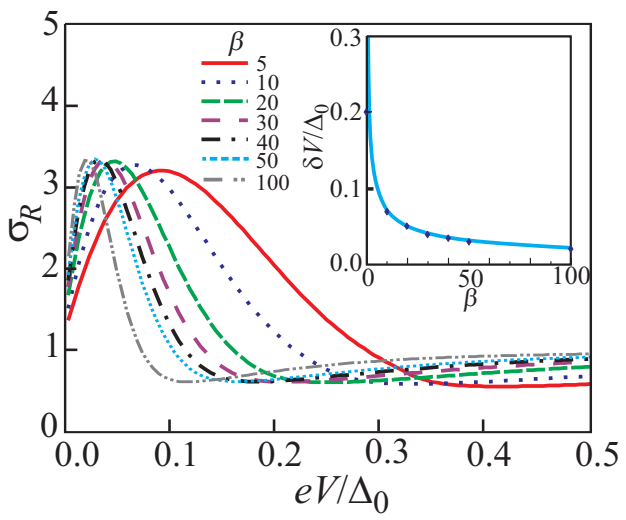

FIG. 2: Relative differential conductance $\sigma_{R}$ for different values of $\beta$ with $Z=3$, the inset shows how the maximum of $\sigma_{R}$ changes with $\beta$.

$\delta V=\left\langle\left|V_{A}\right|\right\rangle=e \hbar \lambda H\langle|\sin \theta|\rangle / m$. For $\beta>>1, \delta V$ is approximately

$$
\delta V=\left\langle\left|V_{A}\right|\right\rangle=\Delta_{0} \frac{H}{H_{c}} \frac{1}{\sqrt{\pi \beta}} .
$$




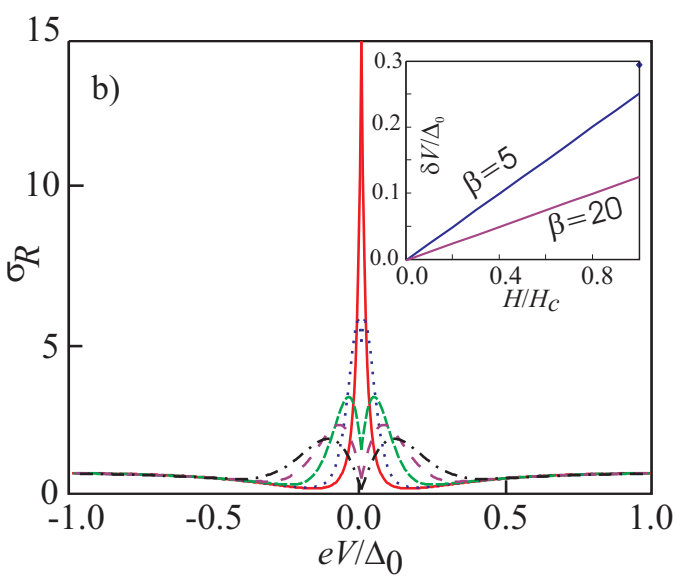

FIG. 3: $\sigma_{R}$ for different values of $H$ with $Z=3$, the inset shows how the peak of $\sigma_{R}$ changes with $H$.

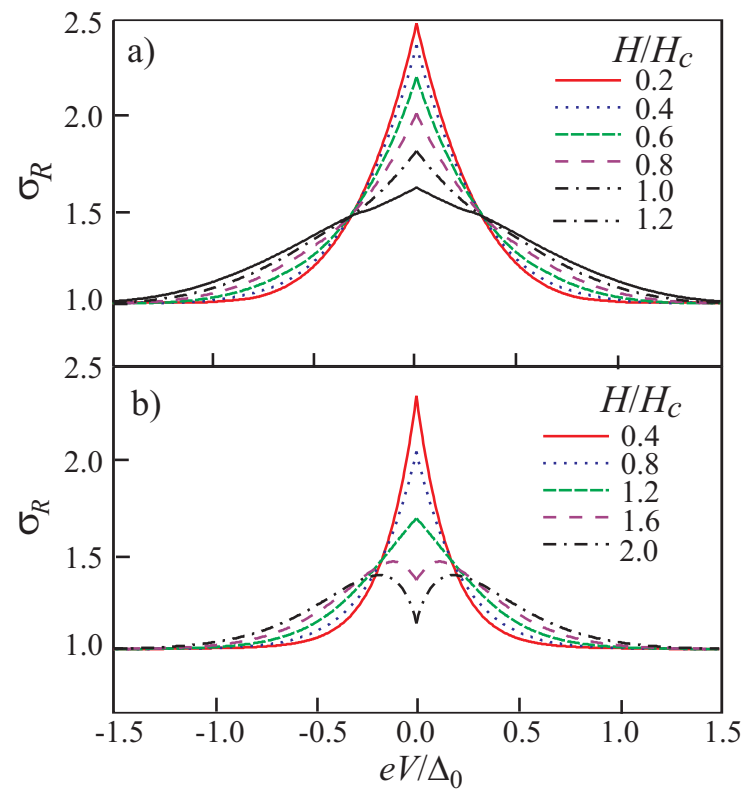

FIG. 4: $\sigma_{R}$ for different values of $H$ with $Z=0.5$, (a) $\beta=5$, (b) $\beta=$ 10.

The average shift $\delta V$ is strongly affected by $\beta$, this behavior is due to the fact that as $\beta$ increases, the tunneling cone diminishes and therefore the shift energy decreases. Fig. 2 shows $\sigma_{R}$ at different values of $\beta$, the splitting of ZBCP increases as $\beta$ decreases. In the inset of the Fig. 2 it is contrated the average shift obtained in Eq. (14) with the numerically value. Fig. 3 shows $\sigma_{R}$ for different values of $H$ with $Z=3$, ZBCP is split and the shift depends on the magnetic field and $\beta$, as shown the inset of Fig. 3. Fig. 4(a) shows $\sigma_{R}$ for different values of $H$ with $Z=0.5$. Is observed that beyond some value the magnetic field ( $\left.H_{\min }\right)$ the ZBCP is split. This value depends on $Z$ and $\beta$ as shown the figures 4(a) and 4(b). For an estimation of $H_{\text {min }}$ one can compare $\delta V$ with the width $\Gamma_{0}$ of the ZBCP. For an angle $\theta$, the width is given by

$$
\Gamma_{0}(\theta)=\frac{T_{N}|\Delta(\theta)|}{2\left(1-T_{N}\right)^{1 / 2}} .
$$

Averaging over $\theta$ the width is approximately

$$
\bar{\Gamma}_{0}=\left\langle\Gamma_{0}(\theta)\right\rangle \simeq \frac{2 \Delta_{0}}{Z\left(1+Z^{2}\right)^{1 / 2} \sqrt{\pi \beta}} .
$$

As $\beta$ increases , $\bar{\Gamma}$ decreases, this is because the tunneling cone decreases and therefore average gap diminishes. The splitting appears when $\delta V \sim \bar{\Gamma} / 2$, from this relation we obtained $H_{\min }$

$$
H_{\min }=\frac{H_{c} p}{Z\left(1+Z^{2}\right)^{1 / 2}},
$$

with $p \sim 1$. $H_{\min }$ is independent of $\beta$, this is due to the fact that as $\beta$ increases, the width of ZBCP decreases but also $\delta V$ decreases and these effects compensate each other. It is important to stand out that although in this case $H_{\min }$ is independent of $\beta, H_{\max }$ depends on $\beta$. If $H>H_{\min }$ the magnetic field induces a splitting of the ZBCP, but if $H_{\min }>H_{\max }$ it is not possible to observe this splitting, as is shown in figures 4(a) and $4(\mathrm{~b})$ for $\beta=5$ and $\beta=20$ respectively, and $H_{\min } \simeq 1.4 H_{c}$ with $p=0.8$. If $\beta=5, H_{\max }=1.2 H_{c}<H_{\min }$ and the splitting does not take place. If $\beta=20, H_{\max } \simeq 2.2 H_{c}$ and $\mathrm{ZBCP}$ is split. In both cases if $H<H_{\min }$ the effect of the magnetic field is to decrease the height of the ZBCP.

Now we study the effect of the Fermi energy difference between the normal and superconductor regions. This difference is quantified by the parameter $\chi=k_{F S} / k_{F N}$, the average angle in Eq. (10) is modified by

$$
\langle\ldots\rangle=\frac{\int_{-\theta_{m}}^{\theta_{m}} d \theta_{S} e^{-\beta \chi^{2} \sin ^{2} \theta_{S}} \cos \theta_{S}}{\int_{-\theta_{m}}^{\theta_{m}} d \theta_{S} e^{-\beta \chi^{2} \sin ^{2} \theta_{S}} \cos \theta_{S} \ldots} .
$$

where $\theta_{m}$ is the maximum angle in the superconductor, for $\chi<$ $1, \theta_{m}=\pi / 2$ and for $\chi>1, \theta_{m}=\sin ^{-1}(1 / \chi)$. If $\beta \chi^{2}>>1$ from Eq's. (10), (14), (15), $\delta V$ and $\bar{\Gamma}_{0}$ are

$$
\begin{aligned}
& \delta V=\Delta_{0} \frac{H}{H_{c}} \frac{1}{\sqrt{\pi \beta} \chi} \\
& \bar{\Gamma}_{0} \simeq \frac{4 \Delta_{0}}{\sqrt{\pi \beta\left(1+\chi^{2}+4 Z^{2}\right)\left((1-\chi)^{2}+4 Z^{2}\right)}} .
\end{aligned}
$$

In this case $H_{\min }$ and $H_{\max }$ are

$$
\begin{aligned}
& H_{\min } \simeq \frac{4 \chi H_{c} p}{\sqrt{\pi \beta\left(1+\chi^{2}+4 Z^{2}\right)\left((1-\chi)^{2}+4 Z^{2}\right)}}, \\
& H_{\max } \simeq \frac{\chi H_{c} \sqrt{\beta}}{2.15} .
\end{aligned}
$$

In Figs. 5(a) and 5 (b) $\sigma_{R}$ is plotted against $V$ for $\chi=0.5$ and $\chi=1.5$ respectively. From Eq. (22) $H_{\min }=0.8 H_{c}$ and $H_{\text {min }}=1.6 H_{c}$ in agreement with the numerical results shown in the figures.

Finally we study the differential conductance at finite temperature, in this case $\sigma_{R}$ is calculated from

$$
\sigma_{R}(e V)=\int_{-\infty}^{\infty} d E \sigma_{R}(E) \frac{d(f(E-e V)-f(E))}{d(e V)},
$$

with $f(E)$ the Fermi-Dirac distribution function at temperature $T$ : Fig. 6 shows $\sigma_{R}$ for $T=0.1 T_{c}$ and for different values 


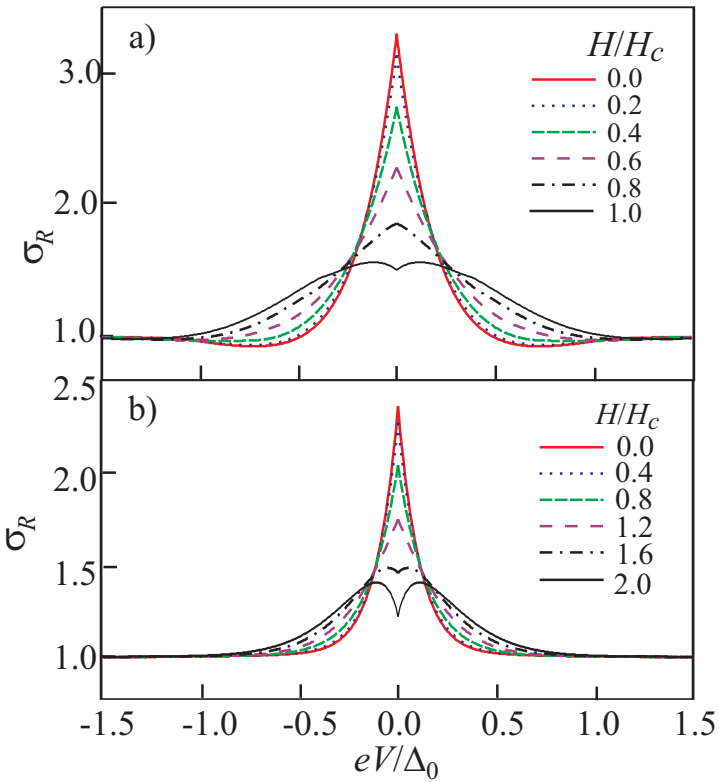

FIG. 5: $\sigma_{R}$ when $E_{F N} \neq E_{F S}$ for different values of $H$. a) $\chi=0.5$, (b) $\chi=1.5$. In both cases $Z=0.5$ and $\beta=20$.

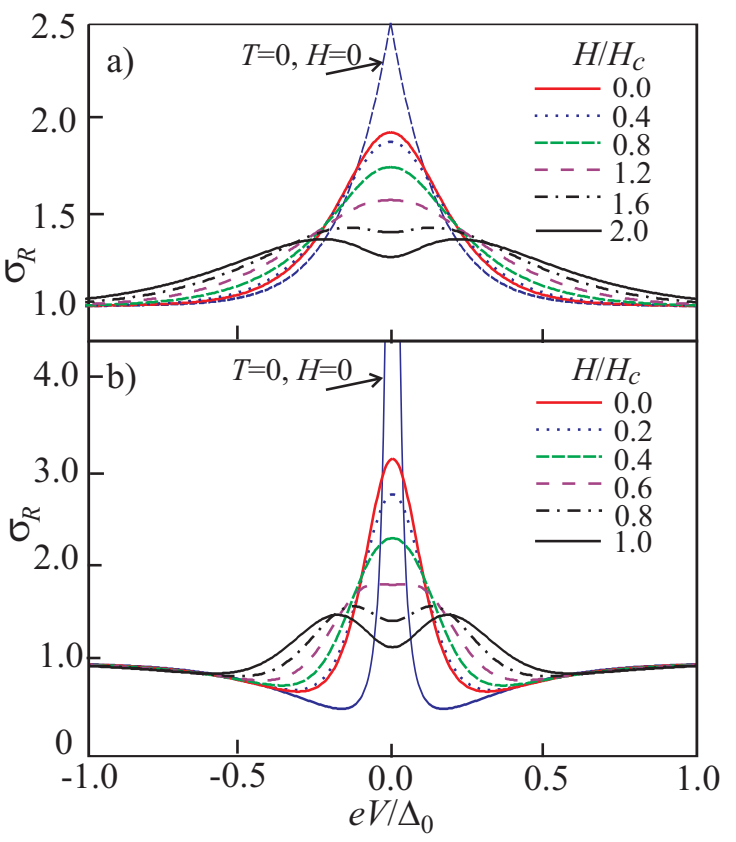

FIG. 6: $\sigma_{R}$ for different values of $H$ with $T=0.1 T_{c}$, (a) $Z=0.5$, (b) $Z=3$. In both cases $\beta=20$ and $\chi=1$. of $Z$. The main effect of $T$ is to increase the width of the peak to $H=0$ and therefore to increase $H_{\min }$. In order to analyze the width of the ZBCP we considerer first the case $k_{B} T<<\bar{\Gamma}_{0}$, we find that width of the $\mathrm{ZBCP}$ is

and $H_{\min }$ is given by

$$
\begin{aligned}
& \bar{\Gamma}(T)=\bar{\Gamma}_{0}\left(1+\frac{3}{2}\left(\frac{K_{B} T}{\bar{\Gamma}_{0} / 2}\right)^{2}+O\left(\frac{K_{B} T}{\bar{\Gamma}_{0} / 2}\right)^{4}\right), \\
& \text { is given by }
\end{aligned}
$$

$$
H_{\min }(T)=H_{\min }(0)\left(1+\frac{3}{2}\left(\frac{K_{B} T}{\bar{\Gamma}_{0} / 2}\right)^{2}\right) .
$$

Therefore the value $H_{\min }$ at finite temperature is larger than $H_{\min }$ for $T=0$. The relative difference $\Delta H / H_{\min }(0)=\left(H_{\min }(T)-\right.$ $\left.H_{\min }(0)\right) / H_{\min }(0)$ is proportional to $K_{B}^{2} T^{2} / \bar{\Gamma}_{0}^{2}$, and therefore in this case the relative increase of $H_{\min }$ is always less than one, as shown in Fig, 6(a) where $H_{\min } \simeq 1.6 H_{c}$; from Eq's. (17) and (22) $H_{\min }(0)=1.4 H_{c}$ and $H_{\min }(T)=1.57 H_{c}$ as shown in the figures. For $k_{B} T>>\bar{\Gamma}_{0}$

$$
\begin{aligned}
& \bar{\Gamma}(T)=1.76 k_{B} T\left(1+O\left(\bar{\Gamma}_{0} /\left(k_{B} T\right)^{2}\right),\right. \\
& H_{\min } \simeq 1.76 k_{B} T \sqrt{\pi \beta} p H_{c} /\left(2 \Delta_{0}\right) .
\end{aligned}
$$

In this case the width is determined main by the temperature $\bar{\Gamma} \sim$ $K_{B} T, H_{\min }(T)$ is proportional to $T$ and $\beta^{1 / 2}$, and can be larger than $H_{\text {min }}(0)$, in contrast to the previous case, see Fig. 6(b).

\section{CONCLUSIONS}

Starting from the solutions of the Bogoliubov of Gennes equations in an NIS junctions we have determined the effect of a magnetic field on the differential conductance. For $d_{x y}$ symmetries the differential conductance presents a ZBCP. The magnetic field induces a splitting of the ZBCP when $H>H_{\min }$, where $H_{\min }$ depends on $Z$, $\chi$, and $T$, in this case the shift of the peak is proportional to $H /\left(\beta^{1 / 2} \chi\right)$. When $H<H_{\min }$ the effect of the magnetic field is to decrease of the height of the ZBCP. If $H_{\min }>H_{\max }$, the splitting does not take place when the magnetic field is applied; the value of $H_{\max }$ depends on $\chi$, and $\beta$. This results can be used for the interpretation of the tunneling characteristic in high Tc superconducting junctions in applied magnetic fields.

Acknowledgments The authors have received support from División de Investigaciones de la Universidad Nacional de Colombia sede Bogotá.
[1] C. C. Tsuei and J. R. Kirtley, Rev. Mod. Phys. 72969 (2000).

[2] M. Covington, M. Aprili, E. Paraoanu, F. Xu, J. Zhu and C. A. Mirkin, Phys. Rev. Lett. 79, 277(1997).

[3] M. Aprili, E. Badica and L. H. Greene, Phys. Rev. Lett. 83, 4630 (1999).

[4] A. Alff, H. Takashima, S. Kashiwaya, N. Terada,H. Ihara,
Y. Tanaka, M. Koyanagi, and K. Kajimura, Phys. Rev. B 55 R14757 (1997).

[5] R. Beck, Y. Dagan, A. Milner, A. Gerber and G. Deutscher, Phys. Rev. B. 69,144506 (2004).

[6] I. Iguchi, W. Wang, M. Yamazaki, Y. Tanaka and S. Kashiwaya, Phys. Rev. B, 62, R6131 (2000). 
[7] S. Kashiwaya, Y . Tanaka, M . Koyanagi, H . Takashima and K. Kajimura, Phys. Rev. B 51, 1350 (1995).

[8] S. Kashiwaya and Y. Tanaka, Rep. Prog. Phys. 63,1641 (2000)

[9] M. B. Walker, P. Pairor, Phys. Rev. B 60, 10395 (1999).

[10] T. Löfwander, V. S. Shumeiko and G. Wendin, Supercond. Sci. Technol. 14, R53 (2001).

[11] M. Fogelström, D. Rainer and J. A. Sauls, Phys. Rev. Lett. 79 281 (1997).

[12] Y. Tanaka, H. Tsuchiura, Y. Tanuma and S. Kashiwaya, J. Phys.
Soc. Jpn. 71 271, (2002).

[13] J. W. Ekin, Yizi Xu, S. Mao, T. Venkatesan, D. W. Face, M. Eddy and S. A. Wolf. Phys. Rev. B 56, 13746 (1997).

[14] A. Biswas, P. Fournier, M. M. Qazilbash, V. N. Smolyaninova, H. Balci, and R. L. Greene, Phys. Rev. Lett. 88, 207004 (2002).

[15] C. Bruder, Phys. Rev. B 41, 4017 (1990).

[16] G. E. Blonder, M. Tinkham and T. M. Klapwijk, Phys. Rev. B 25, 4515 (1982). 